Nathalie Bauer, Isabella Buck

\title{
"nur dass sie_s mal geHÖRT ham;“ - Eine Konstruktion zum Zwecke des Wissensmanagements in medizinischen Interaktionen
}

\begin{abstract}
Häufig stehen ÄrztInnen im Klinikalltag vor der Aufgabe, ihren PatientInnen sowohl Wissen zu vermitteln, das im Hier und Jetzt unmittelbar relevant ist, als auch Wissensangebote $\mathrm{zu}$ machen, deren Bedeutung für die PatientInnen möglicherweise erst zu einem künftigen Zeitpunkt zum Tragen kommt. Dabei bedienen sich ÄrztInnen bisweilen einer matrixsatzlosen „nur dass Sie“-Konstruktion. Anhand detaillierter sequenzanalytischer Betrachtungen des interaktiven Gebrauchs solcher Finalkonstruktionen in einem Korpus von Arzt-Patient-Gesprächen wird argumentiert, dass es sich hierbei insofern um eine hochfunktionale Ressource für das institutionelle Wissensmanagement handelt, als ÄrztInnen durch sie die Situationsrelevanz eingebrachter Informationen modalisieren können.
\end{abstract}

Keywords: Medizinische Gesprächsanalyse, Interaktionale Linguistik, Wissensmanagement, Finalkonstruktion

\section{Einleitung}

Im medizinischen Alltag sehen ÄrztInnen sich nicht selten mit der Herausforderung konfrontiert, PatientInnen mit einer Vielzahl an Informationen zu versorgen, die für deren aktuelle Situation jedoch mitunter von sehr unterschiedlicher Relevanz sind. In solchen Kontexten greifen medizinische ExpertInnen immer wieder auf die im Fokus dieses Aufsatzes stehende Konstruktion nur dass Sie zurück: Wie im Folgenden aufgezeigt wird, werden so bestimmte Informationen in ihrer unmittelbaren Situationsrelevanz restringiert und als Wissensangebote für ein mehr oder weniger wahrscheinlich eintretendes Zukunftsszenario gerahmt. Die Analyse von 20 Belegen aus onkologischen und palliativmedizinischen Arzt-Patient-Gesprächen

Nathalie Bauer, Westfälische Wilhelms-Universität Münster, Germanistisches Institut, nathalie.bauer@uni-muenster.de

Isabella Buck, Westfälische Wilhelms-Universität Münster, Germanistisches Institut, isabella.buck@uni-muenster.de

Ә Open Access. ( 2021 Nathalie Bauer et al., publiziert von De Gruyter. (c) BY-NC-ND Dieses Werk ist lizenziert unter einer Creative Commons Namensnennung - Nicht-kommerziell - Keine Bearbeitung 4.0 International Lizenz. https://doi.org/10.1515/9783110688696-006 
hat gezeigt, dass dieses verfestigte sprachliche Muster eine wichtige Ressource für das institutionelle Wissensmanagement im Krankenhaus darstellt.

Dass die hier zu betrachtenden Finalsätze ohne voran- oder nachgestellten Matrixsatz auftreten, deutet nicht etwa auf eine vermeintlich der Mündlichkeit geschuldete ,Unvollständigkeit‘ hin, sondern stellt ein entscheidendes Charakteristikum der Konstruktion dar: Die vorliegenden dass-Sätze bezeichnen - anders als mit dass eingeleitete Finalsätze im herkömmlichen Sinne (z. B. „Zieh dich warm an, dass du später nicht frierst!") - kein Ziel, auf das die im Verbalkomplex eines übergeordneten Matrixsatzes bezeichnete Tätigkeit ausgerichtet ist (vgl. Dürscheid 2010: 60), sondern (re-)qualifizieren eine gesamte Äußerung in Bezug auf die Mittelbarkeit ihrer situativen Relevanz. Die vorliegende Konstruktion kann folglich als sprechhandlungsbezogene Finalkonstruktion beschrieben werden. ${ }^{1}$

Bevor die beiden prototypischen Verwendungen der Finalkonstruktion anhand der sequenzanalytischen Rekonstruktion dreier Beispielausschnitte exemplarisch aufgezeigt werden, soll zunächst die vorliegende nur dass Sie-Konstruktion von bisherigen grammatischen Beschreibungen (insubordinierter) dass-Sätze abgegrenzt und das zugrunde liegende Konzept des interaktiven Wissensmanagements reflektiert werden.

\section{Freistehende und pseudo-eingebettete dass-Konstruktionen}

Die im Zentrum des Interesses stehende Konstruktion ${ }^{2}$ weist im Vergleich zu den traditionell in Grammatiken behandelten dass-Sätzen syntaktisch die Besonderheit auf, dass der auf die Abtönungspartikel nur folgende dass-Satz ohne Matrixsatz auftritt. Solche alleinstehenden dass-Sätze wie beispielsweise Exklamativ- oder Wunschsätze („Dass bloß morgen die Sonne scheinen möge“; Altmann 1987: 36-38) behandelt die einschlägige Forschungsliteratur nur marginal, wobei sie entweder als „isolierte“ (Buscha 1976: 274) oder „freie“ Nebensätze (Reis 1997: 135) beschrieben, mitunter aber auch als selbstständige Hauptsätze klassifi-

1 Dieser Terminus wurde in Analogie zum Begriff der sprechaktbezogenen weil-Sätze (Günthner 1993: 40) gewählt.

2 In Anlehnung an ein gebrauchsbasiertes Verständnis von Konstruktionen als musterhaften Verfestigungen (vgl. Günthner 2011; Imo 2015) wird hier und im Folgenden mit dem Begriff Konstruktion auf rekurrent auftretende Form-Funktions-Paare referiert, die Interagierende für spezifische kommunikative Handlungen nutzen und die nicht-kompositional sind (vgl. Goldberg 1996: 68-69). 
ziert werden (vgl. Weuster 1983: 63f.). Neben der syntaktischen Ebene zeichnen sich die bislang in der Forschung betrachteten isolierten dass-Nebensätze auch auf der semantisch-pragmatischen Ebene durch Selbstständigkeit aus. Oppenrieder (1991: 177) bringt dies in seiner Benennung der entsprechenden Strukturen als „illokutiv völlig selbstständige Verb-Letzt-Sätze“ zum Ausdruck. Insgesamt wird den alleinstehenden dass-Syntagmen ein autarkes semantisch-pragmatisches Funktionsspektrum zugestanden.

Dass die Gebrauchsweisen der isolierten dass-Konstruktionen jedoch weit über diesen eng gefassten, zudem allein anhand von (konstruierten) schriftsprachlichen Belegen umrissenen Bereich hinausgehen, zeigt Günthner (2013, 2014), die in ihren interaktionallinguistisch-konstruktionsgrammatisch ausgerichteten Untersuchungen eine Vielzahl an Konstruktionstypen von in Alltagsinteraktionen gebräuchlichen dass-Sätzen herausarbeitet. Dabei differenziert sie grundlegend zwischen projizierten, d. h. einen ungesättigten Matrixsatz ergänzenden, und expandierenden dass-Segmenten. Außerdem geht Günthner (2013: 237) auch auf dass-Konstruktionen ein, bei denen eine Bezugssequenz gänzlich fehlt, und beschreibt diese als Folge einer „Relevanzabstufung des Matrixsatzes bei gleichzeitiger pragmatischer Aufwertung und verstärkten Gewichtung des dass-Syntagmas“. Diese Aufwertung manifestiert sich schließlich auch darin, dass das dass-Segment als prosodisch eigenständige Einheit realisiert wird. Insgesamt zeigen Günthners (2013: 228) Ausführungen, dass die „gängige Dichotomie standardsprachlicher Annahmen von Haupt- und Nebensatz in Frage“ zu stellen ist und sich die Integration zwischen dass-Segment und Bezugssyntagma als graduelles Phänomen erweist. Dies resultiert aus der prozesshaften, emergenten Natur gesprochener Sprache, weshalb es sich bei den normgrammatisch nicht fassbaren, isolierten dass-Konstruktionen keineswegs um deviante Strukturen handelt, sondern um funktionale Gebilde, die als Ressourcen zur Realisierung spezifischer kommunikativer Aufgaben eingesetzt werden (vgl. Günthner 2014: 191).

Trotz der zunächst auf der sprachlichen Oberfläche auftretenden formalen Ähnlichkeit der von Günthner beschriebenen mit den im vorliegenden Beitrag behandelten dass-Syntagmen ergeben sich bei näherer Betrachtung zwei gewichtige Unterschiede: Zum einen handelt es sich bei den von Günthner untersuchten Konstruktionen um Inhaltssätze, d. h. „mit dem entsprechenden Satz [wird] nur ein Inhalt transportiert [...] und nicht gleichzeitig ein spezifisches semantisches Verhältnis“ (Eisenberg 2013: 315). Im Gegensatz dazu verfügt die Konjunktion dass in den nur dass Sie-Konstruktionen über eine finale Semantik, womit keine Inhaltssätze, sondern (sprechhandlungsbezogene) Finalkonstruktionen vorliegen. 
Zum anderen treten die in diesem Beitrag zu analysierenden dass-Sätze nicht vollständig isoliert auf, sondern werden zumindest von der Abtönungspartikel nur eingebettet. Während Buscha (1976: 277) die Möglichkeit einer solchen Struktur zwar thematisiert, in ihrem Korpus jedoch „keine die einleitende Konjunktion stützenden Partikeln“ findet, setzt Schlobinski (1992: 205-254) hierfür den zwischen eingebetteten und nicht eingebetteten dass-Sätzen stehenden Typ der pseudo-eingebetteten dass-Sätze an („,bloß daß wir keen wort französisch konntn“). Auf Grundlage eines Korpus psychotherapeutischer Interaktionen beschreibt er diese Konstruktion als eine von den TherapeutInnen eingesetzte Ressource zur Herstellung von Empathie bei gleichzeitiger Zurückstufung des Therapeuten-Ich, was durch den Verzicht auf einen expliziten Einstellungsoperator wie „Ich glaube/meine“ möglich wird. Bei der einleitenden Partikel handle es sich insofern um einen „Dummy“, als sie einen Ersatz für den fehlenden Matrixsatz darstelle (Schlobinski 1992: 253). Im vorliegenden Beitrag soll die Partikel nur hingegen nicht als Dummy betrachtet werden, da eine solche Sichtweise dem ohnehin persistenten „written language bias“ (Linell 2005) weiter Vorschub leistet. Stattdessen wird sie als fester Bestandteil der Konstruktion angesehen, der zu ihrer spezifischen Semantik und Pragmatik beiträgt.

Zusammenfassend lässt sich in Bezug auf die zu untersuchende nur dass Sie-Konstruktionen Folgendes festhalten: Wenngleich diese prima facie zwar als subordiniert erscheinen, da sie die typischen syntaktischen Merkmale wie einleitende Konjunktion in linker Satzklammer und Verbendstellung aufweisen, handelt es sich um sowohl prosodisch als auch semantisch-pragmatisch insubordinierte Strukturen (vgl. Evans 2007: 367).

Da die nur dass Sie-Konstruktion in diesem Beitrag hinsichtlich ihrer interaktiven Funktion beschrieben wird, PatientInnen Wissensangebote für mögliche Zukunftsszenarien zu unterbreiten, soll nachfolgend zumindest skizzenhaft auch das zugrunde gelegte Verständnis von Wissen in der Interaktion dargelegt werden.

\section{Wissensmanagement in der Interaktion}

Wissen stellt eine alltagsweltlich ubiquitäre Größe dar und ist als solche auch in Interaktionen zwischen VertreterInnen von Institutionen und deren KlientInnen omnipräsent. Aufgrund der „Intransparenz des Fremdbewusstseins“ (Bergmann \& Quasthoff 2010: 22) lässt es sich jedoch nicht direkt und unvermittelt beobachten, sodass kaum Aussagen über den de facto vorhandenen Wissensbe- 
stand von Individuen getroffen werden können. Dieses methodologische Problem kann aus der sozialkonstruktivistisch-gesprächsanalytischen Sichtweise, der sich dieser Beitrag verpflichtet sieht, allein durch einen Perspektivwechsel gelöst werden: Wissen wird innerhalb dieses Paradigmas nicht als mentales Konstrukt verstanden, sondern als dynamische, sich in der Interaktion konstituierende Größe (vgl. Deppermann 2018; Heritage 2013). Das Wissensmanagement zwischen Interagierenden kann folglich nicht allein als eine unilaterale Beanspruchung und Zuschreibung von Wissen konzeptualisiert werden; vielmehr ist es als sequenziell verankerte Herstellung von Intersubjektivität in Bezug auf bestimmte Wissensaspekte zu begreifen. Die damit verbundenen Verstehensdarstellungen und -aushandlungen betreffen jedoch „nicht nur [...] Fragen der (Ko-)Referenzherstellung, des Verstehens von Begriffen (Intensionen) und Äußerungsbedeutungen“ (Deppermann 2010: 364), sondern darüber hinaus auch - wie in den folgenden Analysen gezeigt werden soll - Fragen der (relativen) Situationsrelevanz bestimmter Wissensaspekte. Insbesondere in medizinischen Gesprächen, die sich durch eine hohe Informationsdichte auszeichnen, vermögen solche Relevanzrestriktionen als eine spezifische Form der Verstehensanleitung einen wesentlichen Beitrag zum Wissensmanagement und damit zur institutionellen Handlungsprogression zu leisten.

\section{Datengrundlage}

Die der Analyse zugrundliegenden Daten entstammen zum einen dem Projekt „Von der Pathologie zum Patienten: Optimierung von Wissenstransfer und Verstehenssicherung“ (Bentz et al. 2017). Das Projektkorpus setzt sich aus 56 onkologischen Aufklärungsgesprächen zusammen, die in den Jahren 2015 und 2016 am Städtischen Klinikum Karlsruhe erhoben wurden. Dabei handelt es sich um Gespräche, in denen PatientInnen nach erfolgter Gewebeuntersuchung erstmalig mit ihrer Krebsdiagnose konfrontiert werden. Neben der Diagnosemitteilung ist auch die Besprechung des Therapieplans mit den PatientInnen (sowie ggf. deren Angehörigen) Gegenstand der Interaktion.

Zum anderen wurden Daten aus dem Projekt „Kommunikation in der Palliativmedizin: Pflegerisches und ärztliches Sprechen mit PalliativpatientInnen“ verwendet. Das für diesen Beitrag zusammengestellte Subkorpus umfasst 71 Visitengespräche zwischen ÄrztInnen und PatientInnen, die in den Jahren 2018 und 2019 auf der Palliativstation des Städtischen Klinikums Karlsruhe aufgezeichnet wurden. Beim Großteil der Gespräche handelt es sich um triadische Interaktionen zwischen ÄrztInnen, PatientInnen und deren Angehörigen im Rahmen 
der täglichen Visite. Die dort besprochenen Themen sind vielfältig und reichen von der Planung der weiteren Versorgung über die Besprechung der Medikation hin zur Thematisierung des Eintritts der Sterbephase. Die Daten beider Projekte wurden nach GAT 2 (Selting et al. 2009) transkribiert.

\section{Sprechhandlungsbezogene Finalkonstruktionen in medizinischen Interaktionen}

Der Untersuchung liegen insgesamt 20 Belege für die nur dass Sie-Konstruktion aus beiden Korpora zugrunde. Neben der optionalen Abtönungspartikel nur ${ }^{3}$ stellen die final gebrauchte Konjunktion dass sowie das Höflichkeitspronomen Sie feste Bestandteile der hier im Fokus stehenden Konstruktion dar. Das jeweils verwendete Verb variiert jedoch: Mit insgesamt neun Belegen mit wissen, fünf Belegen mit der Perfektform gehört haben und zwei Belegen mit der festen Wendung Bescheid wissen tritt die Konstruktion in den vorliegenden Daten am häufigsten mit mentalen Verben auf. Darüber hinaus wird die Konstruktion auch mit emotiven Verbalphrasen wie sich wundern oder enttäuscht sein gebildet. Häufig erscheinen die Finalkonstruktionen in Kombination mit Abtönungspartikeln wie schon, mal und halt. In der Analyse sollen zunächst die Konstruktionen mit mentalen Verben in den Fokus rücken, ehe in Kapitel 5.2 sodann auf die Konstruktionen mit emotiven Verben bzw. Verbalphrasen eingegangen wird.

\subsection{Finalkonstruktionen mit mentalen Verben}

Die Patientin (PAw) ${ }^{4}$ im folgenden Gesprächsausschnitt leidet trotz umfangreicher medikamentöser Behandlung unter Schmerzen und starker Übelkeit. Unmittelbar vor diesem Ausschnitt sprechen Ärztin und Patientin über die Tatsache, dass die Patientin sich momentan aufgrund der umfassenden Medikation mehrmals täglich übergeben muss. Die Ärztin bietet ihr daher indirekt eine palliative Sedierung an, d.h. die Gabe stark beruhigender Medikamente in der Sterbephase.

3 Die finale Konjunktion damit tritt im Korpus nicht mit der Abtönungspartikel nur auf. 4 Die Sprechersiglen für Ärztinnen lauten $A R w$ und für Ärzte entsprechend $A R m$. Analog dazu werden PatientInnen mit $P A m$ und $P A w$, Angehörige mit $A N m$ und $A N w$ abgekürzt. 
Beispiel 1 (Palliativ_24_02_A_20180706)

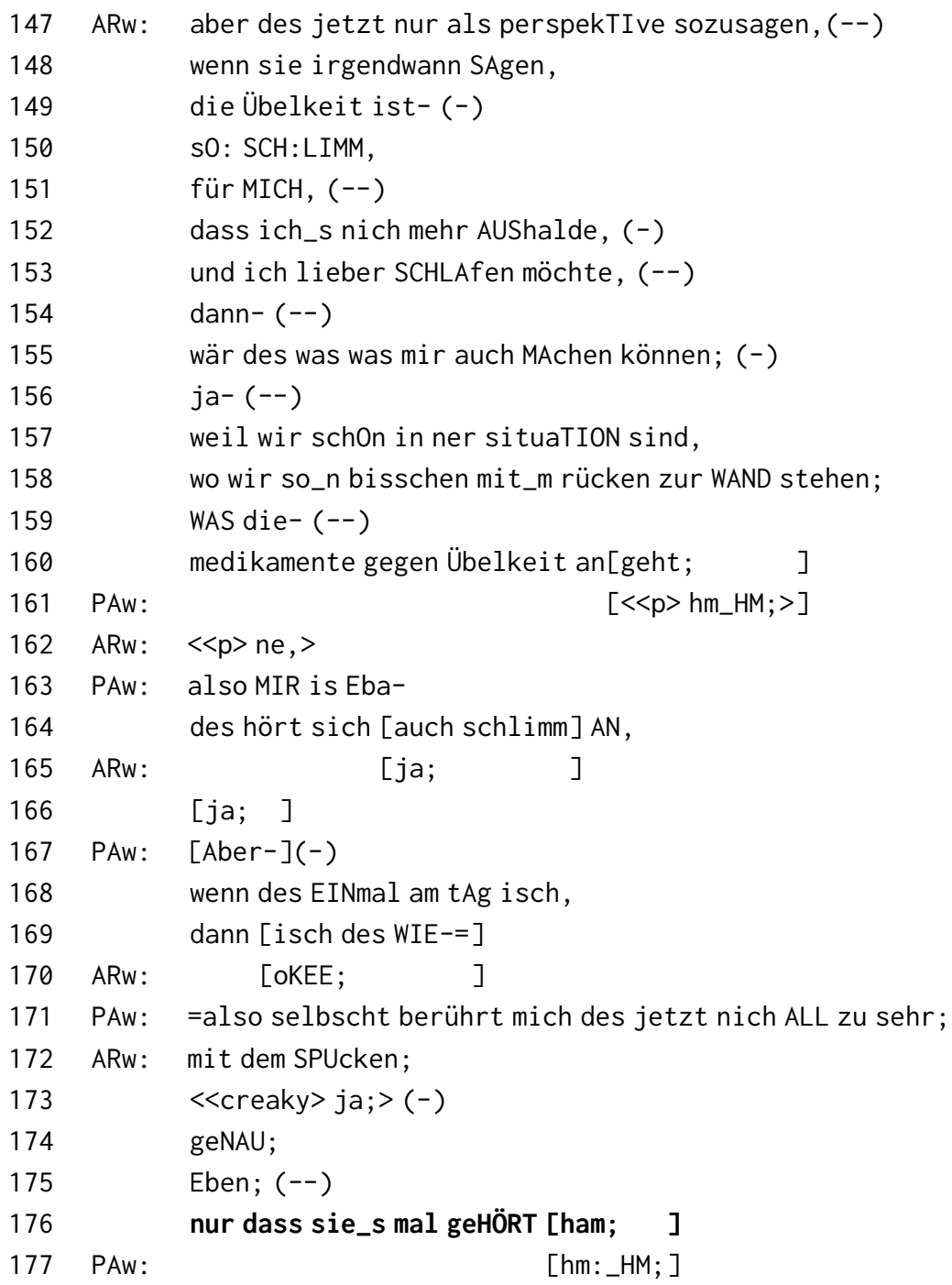

Gleich zu Beginn des Ausschnitts rahmt die Ärztin ihre folgenden Äußerungen prospektiv „nur als perspekTIve“ (Z. 147) und restringiert damit bereits im Voraus die unmittelbare Situationsrelevanz ihrer Ausführungen zur palliativen Sedierung für das Hier und Jetzt des Visitengesprächs. Durch das folgende Konditionalgefüge (Z. 148-155) skizziert sie sodann ein mögliches zukünftiges Szenario, innerhalb dessen die anzusprechende Maßnahme für die Patientin dennoch rele- 
vant werden könnte. Zu diesem „mentalen Raum“ (mental space; Fauconnier 1994), den sie durch den space builder (Fauconnier 1994: 16) „wenn“ etabliert, baut sie zugleich insofern „epistemische Distanz“ (epistemic distance; Fauconnier 1997: 93) ${ }^{5}$ auf, als sie sich in Bezug auf dessen Eintreten als nicht sicher wissend positioniert: Durch das Indefinitadverb „irgendwann“ (Z. 148) verortet sie den dargestellten Fall des verschlechterten Befindens in einer ungewissen Zukunft, ohne dessen Eintrittszeitpunkt „durch ein Prädikat zu charakterisieren und eine Identifizierung durch den Adressaten zu unterstützen bzw. überhaupt erwarten zu lassen“ (Zifonun, Hoffmann \& Strecker 1997: 43). Im Sinne eines empathischen recipient design wird das potenziell bedrohliche Szenario der Sedierung so zunächst einmal nur als ein hypothetisches eingeführt.

Die Ausgestaltung des eröffneten mentalen Raums geht außerdem mit einer Origoverschiebung einher: Die Ärztin gestaltet ihren szenischen Entwurf durch eine animierte Rede (Ehmer 2011: 62) ${ }^{6}$ aus der Perspektive der Patientin, die durch das verbum dicendi „sagen“ (Z. 148) in der Protasis der Konditionalkonstruktion eingeleitet wird. In der durch den Konnektor „dann“ (Z. 154) eingeführten Apodosis thematisiert sie schließlich die Möglichkeit, einen solchen ,Schlafzustand“ im Falle des dargestellten Szenarios medikamentös herbeizuführen. Die Verwendung des Konjunktivs in Z. 155 zeugt dabei weiterhin von der ,epistemisch agnostischen“ (Stivers et al. 2018: 1342) Haltung der Ärztin gegenüber der Sedierungsmaßnahme. Das Sprechen über die Sedierung kann folglich als ein Therapieangebot charakterisiert werden (vgl. Stivers et al. 2018). Eine verbale Minimalreaktion durch die Patientin erfolgt erst in Z. 161, nachdem die Ärztin einen mit „weil“ eingeleiteten account für die Thematisierung der palliativen Sedierung liefert (Z. 157-160). Nach einem verständnissichernden „ne,“ (Z. 162) durch die Ärztin setzt die Patientin zu einer Relativierung ihrer Beschwerden an, indem sie gewissermaßen die äußere Perspektive auf ihre physische und psychische Verfassung mit ihrer eigenen, inneren Sicht kontrastiert: So konzediert sie zwar zunächst eine potenzielle Einschätzung ihres Zustands von außen (dies wird durch den Gebrauch des kognitiven Verbs „anhören“ deutlich) als „schlimm“ (Z. 164), die sie jedoch durch die folgende mit „Aber“ (Z. 167) eingeleitete Adversativkonstruktion als inkongruent zu ihrem eigenen Empfinden darstellt. Besonders hervorgehoben wird der Kontrastaufbau durch die Versetzung des Adverbs „selbst“ ins Vorfeld der folgen-

\footnotetext{
5 Ehmer (2011: 57) spricht in diesem Zusammenhang auch vom „modalen Status“, der ausdrückt, „welche Möglichkeit oder Wahrscheinlichkeit die Sprecher einem mentalen Raum in Bezug auf den Reality Space zuschreiben.“

6 In Anlehnung an Tannen (2007: 112), die solche fiktiven Redeinszenierungen nicht als „reported speech“, sondern als „constructed dialogue“ bezeichnet, entwickelt Ehmer (2011) den Begriff der „animierten Rede“. Vgl. auch Günthner (1997) zu solchen Redeinszenierungen.
} 
den negierten Reformulierung der Patientin in Zeile 171. Durch die überlappend realisierte Rückmeldung „oKEE;“ (Z. 170) sowie die ko-konstruierte Weiterführung der Patientenaussage mit der Präpositionalphrase „mit dem SPUcken;“ (Z. 172) dokumentiert die Ärztin ihr Verstehen.

Mit den Responsivpartikeln „genau“ und „eben“ in den Zeilen 174 und 175 behandelt sie schließlich die patientenseitige Sicht retrospektiv als selbstevident, als keine im Kontrast zu ihren Ausführungen stehende Information (vgl. Oloff 2017; Betz \& Deppermann 2018) und legitimiert so das psychische und somatische Empfinden der Patientin in dieser schwierigen Situation. Durch die folgende nur dass Sie-Konstruktion in Z. 176, die als eigenständige Intonationsphrase realisiert ist, expliziert sie schließlich den hypothetischen Charakter des Therapieangebots: Das Wissen um die palliative Sedierung hat keine unmittelbare Relevanz für das Hier und Jetzt der Behandlungsplanung, sondern ist lediglich in dem in ungewisser Zukunft liegenden konditionalen Raum für die Patientin relevant. Die Finalkonstruktion ist insofern als sprechhandlungsbezogen zu charakterisieren, als die Ärztin mit ihr explizit den Zweck bzw. das Ziel ihrer vorherigen Äußerungen offenlegt und damit im Sinne eines accounts die „Verständlichkeit und den Normbezug des eigenen Handelns“ (Deppermann 2014: 28) ausweist. Das Thematisieren der palliativen Sedierung wird so, vor allem durch den Gebrauch des kognitiven Verbs „hören“, retrospektiv als eine bloße Wissensübermittlung gerahmt, die keine ad hoc-Entscheidung der Patientin relevant setzt und so einer möglichen patientenseitigen Überforderung vorbeugt. Die Patientin wird auf diese Weise mit der Möglichkeit der einschneidenden und potenziell beängstigenden Maßnahme einer palliativen Sedierung vertraut gemacht und damit auf eine mögliche zukünftige Entscheidung vorbereitet. Zugleich fungiert die Finalkonstruktion auch als sequenzschließende Ressource: Durch sie wird die Thematisierung einer palliativen Sedierung abgeschlossen und Ärztin und Patientin wenden sich im Folgenden weiteren Behandlungsmaßnahmen zu. Die Finalkonstruktion dient so nicht allein der (Re-)Qualifikation von Situationsrelevanz eines bestimmten Wissens, sondern fungiert auch als ein exit device aus der Erklärsequenz und dient der Rückkehr zur ärztlichen Agenda nach der Thematisierung dieser potenziell belastenden Information.

\subsection{Finalkonstruktionen mit emotiven Verben}

In Abgrenzung zu den bisherigen Beobachtungen sollen nun solche sprechhandlungsbezogenen Finalkonstruktionen in den Fokus rücken, die mit emotiven Verben bzw. Verbalphrasen gebildet werden. Anhand zweier Beispiele sollen ihre sequenziellen und funktionalen Spezifika exemplarisch aufgezeigt werden. 
Zunächst wird dazu ein Ausschnitt aus einem Gespräch betrachtet, in dem der Patient im Beisein seiner Lebensgefährtin die Diagnose einer Krebserkrankung im Bereich des Lymphgewebes erhält. Im Kontext der anschließenden Besprechung des Therapieplans betont der Arzt dezidiert, dass eine Heilung nicht anvisiert wird:

Beispiel 2 (Onkologie_019-01_191214)

019 ARm: ${ }^{\circ} \mathrm{h}$ unser ZIEL ist,

020 die KRA:NKheit; (---)

021

in SCHACH zu halten; (2.5)

022 aber der ansatz ist !NICHT! ,

023 die für immer und ewig aus dem körper zu ent [FERnen. ]

024 PAm:

$[<<p>j a ;>]$

025 ARm: was HEIlung bedeutet;

026 PAm: ja;

027 ARm: ja? (--)

028

029

030

031

032

033

034

035

036

037

038

039

040

041 wir sind froh wenn es komplett verSCHWINdet, (--) sie würden aber TROTZdem anschließend, in rEgelmäßigen (-) ABständen nachgesorgt werden? (1.3) ${ }^{\circ}$ hh und, (1.2)

MEIStens is es so, =

045

046

=dass die krAnkheit ! IR! gendwann mal wieder kommt;

ANw: hm_HM;

ARm: ja?

PAm: [hm_HM,

ARm: [das kann scho]n SEIN;

PAm: [ja; ]

ANw: [kann] schon SEIN.

hm_HM;

ARm: nur dass sie (-) nicht entTÄUSCHT sind,

(-) wenn es jetzt WEGgeht, und Irgendwann später KOMMT_S wieder , und sie dann SA:gen, warUm ist das bei mir wieder geKoMmen? (1.3)

048

${ }^{\circ} \mathrm{h}$ das ist NICHTS ungewöhnliches; (--)

unser ZIEL ist es, ihnen lebensZEIT, lebensqualiTÄT, (--)

051 PAm:

krankheitsFREIe zeit zu [geben; ] [hm_HM; ] 
Mit der Projektorkonstruktion (Günthner 2008) zu Beginn des vorliegenden Ausschnitts verdeutlicht der Arzt das Ziel der therapeutischen Maßnahmen, die Erkrankung des Patienten „,in Schach zu halten“ (Z. 021). Nach einer 2,5-sekündigen Gesprächspause expliziert er seine metaphorische Äußerung ex negativo dahingehend, dass es sich bei der Therapie nicht um ein kuratives Verfahren handelt (Z. 022-023) und eine „HEIlung“ (Z. 025) folglich nicht anvisiert wird. Der Patient liefert Rückmeldesignale zu den Ausführungen des Arztes (Z. 024, 026), der darauf zunächst mit einem verständnissichernden „ja?“ (Z. 027) reagiert, ehe er schließlich zu einer Elaboration des Therapieziels und einem möglichen Rezidiv ansetzt (Z. 028-033). Durch das prosodisch markierte Indefinitadverb ,!IR!gendwann“ (Z. 033) verortet der Arzt einen möglichen Wiederauftretenszeitpunkt jedoch auch hier in einem ungewissen in der Zukunft liegenden Zeitraum und baut damit nicht nur temporale, sondern auch epistemische Distanz zu dem mentalen Raum auf (Fauconnier 1997: 93, vgl. Beispiel 1). Es folgt eine Sequenz der Verstehensdarstellung und -sicherung (Z. 034-038), die in einer bestätigenden Wiederholung (Schegloff 1996: 195) der ärztlichen Zusammenfassung „das kann schon SEIN“ (Z. 037) durch die Lebensgefährtin in Z. 039 kulminiert.

Im Anschluss wird schließlich die im Fokus des Interesses stehende Konstruktion „nur dass sie (-) nicht entTÄUSCHT sind“ (Z. 041) als eigenständige Intonationsphrase realisiert, mit der der Arzt auch hier einen account für seine vorangehende Wissensvermittlung liefert. Durch die negierte Konstruktion rahmt er die zuvor übermittelten heiklen und potenziell belastenden Informationen retrospektiv als eine Maßnahme zur Prävention möglichen negativen Erlebens durch den Patienten: Die Aufklärung über ein potenzielles Wiederauftreten der Krankheit soll diesen auf das Szenario eines Rückfalls vorbereiten und so eine potenzielle Enttäuschung abfedern. Die Finalkonstruktion kann also auch hier insofern als sprechhandlungsbezogen charakterisiert werden, als durch sie der Zweck bzw. das Ziel der zuvor getätigten Äußerungen expliziert wird. Damit geht eine Zurückstufung der situativen Relevanz der vermittelten Informationen für das Hier und Jetzt einher: Mit dem Konditionalgefüge (Z. 041-045), dessen Apodosis die Finalstruktur selbst darstellt, skizziert der Arzt einen außerhalb des reality space der laufenden Interaktion liegenden mentalen Raum, innerhalb dessen seine Ausführungen für den Patienten potenziell bedeutsam sind.

Sowohl in Bezug auf die temporale als auch die deiktische Ausgestaltung des Konditionals wird auch hier die Abgrenzung des Hier und Jetzt vom skizzierten mentalen Raum deutlich: Zunächst wird der mentale Raum durch die in der Protasis erneut verwendete indefinite Temporalangabe „Irgendwann später“ (Z. 043, s. auch Z. 040) im Gegensatz zum ,jetzt“ (Z. 042) der aktuellen Therapiebesprechung in einer ungewissen Zukunft verortet (s. Kapitel 5.1). Mit der 
durch das verbum dicendi „sagen“ (Z. 044) eingeleiteten animierten Rede, die durch das Temporaladverb „dann“ (Z. 044) in dem soeben eröffneten konditionalen mentalen Raum verortet wird, erfolgt schließlich auch eine deiktische Abgrenzung vom Hier und Jetzt der laufenden Interaktion: Die ätiologische Frage nach dem „warUm“ (Z. 045) eines möglicherweise auftretenden Rezidivs formuliert der Arzt aus der Perspektive seines Patienten und verlässt damit die hic-nunc-ego-Origo.

Mit der folgenden normalisierenden Stellungnahme spricht der Arzt wieder als medizinischer Experte und bewertet das soeben skizzierte Szenario als „NICHTS ungewöhnliches“ (Z. 046). Die Wissensvermittlung sowie deren Relevanzmodalisierung durch die Finalkonstruktion enden schließlich mit einer Reformulierung der eingangs bereits verwendeten Projektorkonstruktion ,unser ZIEL ist es“ (Z. 047), deren B-Teil in Form einer von Selting (2007) als Listenkonstruktion beschriebenen Drei-Komponenten-Struktur erfolgt: „ihnen lebensZEIT, lebensqualiTÄT, (--) krankheitsFREIe zeit zu geben“ (Z. 048-050). Auch hier fungiert die Finalkonstruktion also sequenzschließend.

Wie auch in dem zuvor analysierten Beispiel wird deutlich, dass die sprechhandlungsbezogene Finalkonstruktion sowohl als account für vorherige Wissensvermittlungen sowie auch als retrospektive Modalisierung der unmittelbaren Situationsrelevanz der thematisierten Informationen fungiert. Das Beispiel 2 unterscheidet sich jedoch insofern von dem zuvor analysierten, als es nicht allein um die Relevanz des Wissens als solches (wie der Option einer bestimmten Behandlungsmöglichkeit in Bsp. 1) für den konstruierten mentalen Raum geht, sondern um eine affektive Reaktion auf dessen mögliches Eintreten: Das Wissen um ein mögliches Rezidiv wird vorliegend als eine Art Präventionsmaßnahme vom Arzt übermittelt, um einem möglichen negativen Erleben, einer unerwarteten Enttäuschung durch den Patienten vorzubeugen. In diesem Zusammenhang können die sprechhandlungsbezogenen Finalkonstruktionen mit emotiven Verbalphrasen - die in den untersuchten Daten im Übrigen stets mit der negierten Zuschreibung von Emotionen negativer Valenz erfolgen - als eine prospektiv operierende Ressource zur Anzeige von Empathie im Sinne einer verstehenden Perspektivübernahme charakterisiert werden.

Im Folgenden soll noch ein weiterer Beleg für diesen emotiv ausgerichteten Subtyp analysiert werden. Konträr zu den vorherigen Beispielen ist die epistemische Distanz der Ärztin zum entworfenen Szenario hier sehr gering, weshalb der eröffnete mentale Raum nicht als hypothetisch bezeichnet werden kann.

Der untenstehende Transkriptausschnitt entstammt der ersten Visite der Oberärztin bei einem Patienten, der seit einer Woche auf der Palliativstation liegt. Im bisherigen Gesprächsverlauf wurde bereits über die schlechten Blutwerte des Patienten und die Wassereinlagerungen in seiner Lunge gesprochen. 
Anschließend fährt die Oberärztin damit fort, ein Dilemma bezüglich der Medikation $\mathrm{zu}$ thematisieren: Der Patient leidet unter Herzproblemen und muss daher eigentlich Herzmedikamente nehmen. Diese würden jedoch seine Niere weiter schwächen, die von einem Tumor befallen und daher in ihrer Funktion ohnehin eingeschränkt ist.

Beispiel 3 (Palliativ_233_13_A_20190307)

001 ARw: die M-

002 medikaMENte die sie noch hAtten:,

$003 \quad f: u ̈ r$ ihr HERZ,

004 die müssen weiter pauSIERT bleiben, =

$005 \quad=$ weil die NIEre nAch wie v0r, (---)

006 ANw: hm_HM;

007 ARw: äh-

008 etwas EINgeschränkt in ihrer funktion is;

009 ANw: hm_HM;

010 PAm: ja,

011 klar,

012 ARw: is nich SCHLECHter geworden,

013 aber auf kEInen fall BESser; ;

$014=<<p>$ also WIR- $>$

$015 \quad{ }^{\circ} \mathrm{h}<<a l l>$ letztes mal hatten wer eins VIER, =

$016 \quad=$ jetzt ham_mer eins FüNF , $>$

$017 \quad<<p>$ das is SO-

018 ANw: [hm_HM, ]

019 ARw: [gleiches] niVEAU; >=

020 ANw: =hm_HM,

$021 \quad<<\mathrm{p}>\mathrm{hm} \_\mathrm{HM}$;

$022 \quad$ [hm_HM; $>$ ]

023 ARw: [aber DANN, ]

024 m: üss mer_s weider pauSIEren; =

025 =nur dass sie sich nich wUndern wo sind meine medikaMENte;

026 ANw: $\quad<$ f > ' n:ee,>

027 PAm: [(hm_HM/nein, ) ]

028 ANw: [<<behaucht $>$ da hätt ich dann sch]on nach geFRA[GT; ]

029 ARw: $\quad[\ll<f j a]$

DüRfen sie ja; 
Während die Ärztin in Beispiel 1 der Patientin ein Angebot hinsichtlich eines spezifischen Medikamentes unterbreitete, formuliert die Oberärztin hier zunächst qua ihrer fachlichen Autorität und Expertise die unilateral getroffene Therapieentscheidung, dass die Herzmedikamente des Patienten „weiter pauSIERT bleiben,“ müssen (Z. 004). Diese Maßnahme wird gegenüber dem Patienten nicht zur Disposition gestellt, sondern durch das deontisch gebrauchte Modalverb „müssen“ (Z. 004) als zwingend und daher als nicht verhandelbar angekündigt. Trotz des fehlenden Angebots einer weiteren Diskussion über die Medikation liefert die Ärztin sodann einen auf fachmedizinischem Wissen basierenden account für ihre Ankündigung (vgl. Angell \& Bolden 2015: 47), indem sie durch die kausale Konjunktion „weil“ eine Erklärung einleitet: „= weil die NIEre nAch wie vOr, (---) [...] äh- etwas EINgeschränkt in ihrer funktiOn is;“ (Z. 005-008). Sowohl die Ehefrau des Patienten als auch der Patient selbst ratifizieren im Folgenden diese Erklärung, ehe die Ärztin mit einer näheren Erläuterung zum Krankheitsverlauf in Form einer Adversativstruktur fortfährt: „is nich SCHLECHter geworden, aber auf kEInen fall BESser;“ (Z. 012-013). Diese Aussage wird sodann weitergehend spezifiziert, indem die Ärztin auf die konkreten Nierenwerte zurückgreift und so für die Entscheidung, die Medikamente abgesetzt $\mathrm{zu}$ lassen, weitere fachliche Evidenz anführt. Im Anschluss daran wiederholt sie die bereits unilateral getroffene Entscheidung und rahmt diese so als aus medizinischen Fakten resultierenden Kulminationspunkt (Z. 023-024). Danach folgt schließlich die prosodisch eigenständig realisierte nur dass Sie-Konstruktion (Z. 025).

Im Gegensatz zu den beiden vorangehend analysierten Beispielen ist die nur dass Sie-Konstruktion hier nicht in einen durch eine Konditionalstruktur eröffneten mentalen Raum eingebettet. Dennoch verweist die Ärztin auch hier prospektiv auf ein in der Zukunft, d. h. außerhalb des Hier und Jetzt (reality space) liegendes Szenario, das sich durch das Fehlen der Herzmedikamente auszeichnet. Wie auch in den anderen beiden Beispielen geht die Ausgestaltung dieses Szenarios mit einer verbalisierten Perspektivübernahme in Form einer Origoverschiebung einher: Durch die Aussage „wo sind meine medikaMENte;“ (Z. 025) verlässt die Ärztin die unmittelbar gegebene deiktische Origo und verankert den in der animierten Rede ausgedrückten Gedanken in der Origo des Patienten.

Dass die Oberärztin an dieser Stelle Wissen vermittelt, das aktuell nicht von unmittelbarer Relevanz ist, liegt in den institutionellen Gegebenheiten begründet: Die Oberärztin kommt lediglich einmal pro Woche zur Visite, sodass sie den Patienten zu diesem Zeitpunkt mit all den Wissensbeständen ,versorgen' muss, die in den folgenden Tagen bis zur nächsten Visite potenziell relevant werden könnten. So nimmt sie auch hier eine Relevanzmodalisierung der 
vermittelten Informationen vor, antizipiert zugleich ein zukünftiges Szenario, in dem die gegebene Information bedeutsam ist, und verhindert so negatives Erleben. Die sprechaktbezogene Finalkonstruktion nur dass Sie sich nicht wundern fungiert hier folglich als eine Art retrospektiver epistemischer Disclaimer (Hewitt \& Stokes 1975), der sich wie folgt paraphrasieren lässt: „Wenn Sie feststellen, dass Ihre Herzmedikamente fehlen, dann erinnern Sie sich an die Information, die ich Ihnen gegeben habe, sodass Sie sich nicht wundern“.

Anders als im vorherigen Beispiel verfügt die Ärztin im vorliegenden Ausschnitt jedoch über eine höhere epistemische Sicherheit bezüglich der Zutreffenswahrscheinlichkeit des mentalen Raums: Sie vermittelt kein Wissen für eine möglicherweise eintretende zukünftige Situation, sondern für ein Szenario, dessen Eintreten sie selbst veranlasst hat. Die epistemische Distanz zu der thematisierten Situation ist folglich - anders als in Beispiel 1 und 2 - sehr gering.

Mit dieser veränderten Wissenskonstellation geht eine institutionsspezifische Funktionserweiterung der Konstruktion einher: Der sprechhandlungsbezogene Finalsatz dient hier nicht allein der Relevanzmodalisierung einer Informationssequenz, sondern vielmehr auch der Rahmung einer dargestellten medizinischen Maßnahme als unverhandelbar. Konträr zu den zuvor analysierten Transkriptausschnitten wird in diesem Beispiel neben der epistemischen Autorität der Ärztin also auch ihre deontische Autorität relevant gesetzt. ${ }^{7}$ Die Ausführung deontischer Autorität liegt in diesem Fall darin, dass sie ohne vorherige Rücksprache mit dem Patienten, sondern allein qua ihres Wissens über die Wechselwirkung zwischen den Herzmedikamenten und der tumorbefallenen Niere die Herzmedikamente abgesetzt hatte. Diese unilateral getroffene Medikationsentscheidung wird durch die Finalkonstruktion als eine Maßnahme gerahmt, über die der Patient sich zukünftig zu wundern vermag - deren Diskussion in der aktuellen Gesprächssituation jedoch nicht zur Disposition gestellt wird. Allerdings handelt es sich bei deontischer Autorität, zumindest einem gesprächsanalytischen Verständnis nach, nicht um eine unilateral hervorgebrachte, seinen InteraktionspartnerInnen ,übergestülpte“ Größe. Vielmehr muss die von einem Sprecher eingenommene deontisch superiore Haltung von den anderen GesprächsteilnehmerInnen ratifiziert werden, um eine relevante Gesprächsgröße darzustellen (vgl. Lukes 1978: 649). Im Falle des vorliegenden Transkriptausschnittes findet eine solche Ratifikation nicht statt: Die Ehefrau des Patienten weist die von der Ärztin vorgenommene Fremdpositionierung ihrer eigenen Person und die ihres Mannes als reine ,Empfänger‘ von Wissen zu-

7 Das Konzept der deontics nimmt innerhalb der gesprächsanalytischen Forschung auf den Anspruch bzw. die Berechtigung von Interagierenden Bezug, künftige Handlungen ihrer GesprächspartnerInnen zu bestimmen (vgl. Stevanovic \& Peräkylä 2012: 299). 
rück und nimmt sprachlich eine deontisch gleichberechtigte Haltung ein: Auf ein prosodisch sehr auffällig konturiertes „,<<> “n:ee,>“ (Z. 026) folgt die Aussage „da hätt ich dann schon nach geFRAGT;“ (Z. 028), mittels derer die Ehefrau innerhalb des von der Ärztin eröffneten Szenarios eine durch den Konjunktiv als hypothetisch gerahmte Reaktion entwirft. Durch das Adverb „da“, dem hier sowohl eine lokale als auch eine konditionale und temporale Semantik zukommt, verankert sie ihre Aussage ebenfalls in diesem hypothetischen künftigen Szenario und verweist darauf, dass sie sich gar nicht erst ,gewundert', sondern sich direkt nach dem Grund für das Absetzen der Medikamente erkundigt hätte. Die Ärztin wiederum positioniert sich im Folgenden abermals als deontisch höherstehend, indem sie der Angehörigen das Recht zuspricht, Informationen einzuholen: „<<f >ja DÜRfen sie ja; ““(Z. 029).

Der Unterschied zwischen diesem und den beiden vorangehend analysierten Beispielen liegt, zusammenfassend betrachtet, im Grad der deontischen Autorität der Ärztin und der epistemischen Sicherheit in Bezug auf die entworfene Zukunft: Da die Ärztin selbst ex ante über die medikamentöse Situation verfügte und somit durch ihr außersprachliches Handeln sowohl Gegenwart als auch Zukunft bereits determinierte, ist der modale Status des konstruierten mentalen Raums durch eine hohe Zutreffenswahrscheinlichkeit charakterisiert (vgl. Ehmer 2011: 56). Die Konstruktion wird hier also nicht allein zur Relevanzmodalisierung eines hypothetisch relevanten Wissens eingesetzt, sondern dient zugleich der deontisch superioren Positionierung durch die Ärztin, die ihre Medikationsvorgabe mittels des Finalsatzes als nicht weiter verhandelbar rahmt. Ein weiterer Unterschied zu den anderen beiden Beispielen besteht ferner darin, dass die Interaktionspartnerin der Ärztin, in diesem Fall die Ehefrau des Patienten, die durch die nur dass Sie-Konstruktion etablierte Welt negiert: Da sie die Fremdpositionierung ihres Mannes als passiver Patient und ihrer eigenen Person als passive Angehörige zurückweist, kann die evozierte Reaktion des Wunderns in Bezug auf das Zukunftsszenario gar nicht erst zustande kommen.

\section{Fazit}

In den vorangehenden Analysen konnte gezeigt werden, dass es sich bei der sprechhandlungsbezogenen Finalkonstruktion nur dass Sie um eine Konstruktion im Sinne eines musterhaft verfestigten, rekurrent auftretenden Form-FunktionsPaares handelt. Diese Konstruktion ist dabei insofern auf die epistemischen Erfordernisse der Institution Krankenhaus zugeschnitten, als sie in deren Rahmen eine spezifische Funktion für das Wissensmanagement der Interagierenden er- 
füllt. Nachfolgend sollen nun die wesentlichen Analyseergebnisse zusammengefasst und Gemeinsamkeiten sowie Unterschiede der beiden hier betrachteten formalen Subtypen der Konstruktion abschließend aufgezeigt werden.

Wird der Blick zunächst auf den Kontext gelegt, in dem die nur dass Sie-Konstruktion auftritt, so ist festzuhalten, dass ÄrztInnen dann auf diese Finalkonstruktion zurückgreifen, wenn sie den PatientInnen Informationen ohne unmittelbare Situationsrelevanz vermitteln. Während etwa das Pflegepersonal die PatientInnen im Laufe eines Tages mehrfach aufsucht, findet die ärztliche Visite nur einmal täglich - die Oberarztvisite sogar nur einmal wöchentlich - statt. Die ÄrztInnen sehen sich daher bisweilen mit der Herausforderung konfrontiert, sowohl Wissensangebote zu machen, deren Bedeutung im Hier und Jetzt unmittelbar zum Tragen kommt, als auch solche Wissensbestände $\mathrm{zu}$ transferieren, die die $\mathrm{Pa}$ tientInnen möglicherweise erst zu einem künftigen Zeitpunkt X benötigen. Die Finalkonstruktion stellt in diesem Kontext eine Lösung für das kommunikative Problem dar, den Zeitraum, zu dem die PatientInnen das vermittelte Wissen benötigen, auszuweisen: Die Konstruktion bildet eine Art epistemischen Disclaimer und indiziert so die nur mittelbare Relevanz des Gesagten was dann zumeist auch zu einer Schließung der jeweiligen Wissenssequenz führt. Nur dass Sie geht den entsprechenden Informationen hierbei entweder voran, rahmt diese also prospektiv, oder erscheint nachgestellt und modifiziert in dieser Position die Situationsrelevanz des vermittelten Wissens retrospektiv. Unabhängig von ihrer Position ist zu beobachten, dass die Konstruktion in allen Fällen in ein von den ÄrztInnen konstruiertes hypothetisches Szenario bzw. einen evozierten mentalen Raum eingebettet ist. In den hier analysierten Beispielen geht das Eröffnen eines solchen Raumes stets mit animierter Rede und einem Origowechsel einher; durch ein solches „Demonstrieren der (Sprech-)Handlung einer Figur in einem mentalen Raum“ (Ehmer 2011: 62-63) mit gleichzeitiger Perspektivübernahme wird ein stärkerer Adressatenbezug erreicht, mit dem wiederum eine höhere Involvierung der PatientInnen einhergeht (vgl. Clark \& Gerrig 1990: 793).

Innerhalb der Konstruktion wurde in Abhängigkeit davon, ob die Leerstelle von einem mentalen Verb wie wissen oder von einem emotiven Verb wie wundern gefüllt ist, zwischen zwei Konstruktionsvarianten differenziert. Die Variante nur dass Sie + emotive Verbalphrase dient neben der Restriktion der unmittelbaren Situationsrelevanz einer Information auch der Prävention möglichen negativen Erlebens: Sind die PatientInnen bereits auf ein potenzielles Szenario vorbereitet, fällt die Reaktion darauf möglicherweise weniger heftig aus. Innerhalb der emotiv ausgerichteten Konstruktionsvariante wurde schließlich ein von den beiden anderen Beispielen abweichender Fall herausgearbeitet: Die epistemische Distanz der Ärztin zum entworfenen Szenario ist hier sehr 
gering, da sie selbst durch ihr außersprachliches Handeln das Szenario, innerhalb dessen sie einem negativen Erleben des Patienten vorzubeugen versucht, bereits erschaffen hat. Der eröffnete mentale Raum, auf den der epistemische Disclaimer nur dass Sie Bezug nimmt, muss folglich nicht zwangsläufig hypothetischer Natur sein.

\section{Literatur}

Altmann, Hans (1987): Zur Problematik der Konstitution von Satzmodi als Formtypen. In Jörg Meibauer (Hrsg.), Satzmodus zwischen Grammatik und Pragmatik. Referate anläßlich der 8. Jahrestagung der Deutschen Gesellschaft für Sprachwissenschaft Heidelberg, 22-56. Tübingen: Niemeyer.

Angell, Beth \& Galina B. Bolden (2015): Justifying medication decisions in mental health care: Psychiatrists' accounts for treatment recommendations. Social Science \& Medicine 138, 44-56.

Bergmann, Jörg \& Uta Quasthoff (2010): Interaktive Verfahren der Wissensgenerierung: Methodische Problemfelder. In Ulrich Dausendschön-Gay, Christine Domke \& Sören Ohlhus (Hrsg.), Wissen in (Inter-)Aktion. Verfahren der Wissensgenerierung in unterschiedlichen Praxisfeldern, 21-34. Berlin, New York: De Gruyter.

Bentz, Martin, Martin Binnenhei, Georgios Coussios, Juliana Gruden, Wolfgang Imo, Lisa Korte, Thomas Rüdiger, Antonia Ruf-Dördelmann, Michael R. Schön, Sebastian Stier (2017): Von der Pathologie zum Patienten: Optimierung von Wissenstransfer und Verstehenssicherung. SpIn - Arbeitspapiere Sprache und Interaktion 72, 1-45.

Betz, Emma \& Arnulf Deppermann (2018): Indexing Priority of Position: Eben as Response Particle in German. Research on Language and Social Interaction 51, 171-193.

Buscha, Annerose (1976): Isolierte Nebensätze im dialogischen Text. Deutsch als Fremdsprache 13 (5), 274-279.

Clark, Herbert H. \& Richard J. Gerrig (1990): Quotations as Demonstrations. Language 66 (4), 767-805.

Deppermann, Arnulf (2010): Konklusionen: Interaktives Verstehen im Schnittpunkt von Sequenzialität, Kooperation und sozialer Struktur. In Arnulf Deppermann, Ulrich Reitemeier, Reinhold Schmitt \& Thomas Spranz-Fogasy (Hrsg.), Verstehen in professionellen Handlungsfeldern, 363-384. Tübingen: Narr.

Deppermann, Arnulf (2014): Konversationsanalyse: Elementare Interaktionsstrukturen am Beispiel der Bundespressekonferenz. In Jörg Hagemann \& Sven Staffeldt (Hrsg.), Pragmatiktheorien: Analysen im Vergleich, 19-47. Tübingen: Stauffenburg.

Deppermann, Arnulf (2018): Wissen im Gespräch. In Karin Birkner, Karin \& Nina Janich (Hrsg.), Handbuch Text und Gespräch, 104-142. Berlin, Boston: De Gruyter.

Dürscheid, Christa (2010): Syntax: Grundlagen und Theorien. 6. aktual. Auflage. Stuttgart: Vandenhoeck \& Ruprecht.

Ehmer, Oliver (2011): Imagination und Animation. Die Herstellung mentaler Räume durch animierte Rede. Berlin, New York: De Gruyter. 
Eisenberg, Peter (2013): Der Satz. Grundriss der deutschen Grammatik. Stuttgart, Weimar: Metzler.

Evans, Nicholas (2007): Insubordination and its uses. In Irina Nikolaeva (Hrsg.), Finiteness. Theoretical and Empirical Foundations, 366-431. Oxford: Oxford University Press.

Fauconnier, Gilles (1994): Mental Spaces: Aspects of Meaning Construction in Natural Language. Cambridge: Cambridge University Press.

Fauconnier, Gilles (1997): Mappings in Thought and Language. Cambridge: Cambridge University Press.

Goldberg, Adele E. (1996): Construction Grammar. In Keith Brown \& James E. Miller (Hrsg.), Concise Encyclopedia of Syntactic Theories, 68-71. Oxford: Pergamon.

Günthner, Susanne (1993): „ ... weil - man kann es ja wissenschaftlich untersuchen“ Diskurspragmatische Aspekte der Wortstellung in WEIL-Sätzen. Linguistische Berichte 143, 37-59.

Günthner, Susanne (1997): Stilisierungsverfahren in der Redewiedergabe - Die „Überlagerung von Stimmen“ als Mittel der moralischen Verurteilung in Vorwurfsrekonstruktionen. In Margret Selting \& Barbara Sandig (Hrsg.), Sprech- und Gesprächsstile, 94-122. Berlin, New York: De Gruyter.

Günthner, Susanne (2008): Projektorkonstruktionen im Gespräch: Pseudoclefts, die Sache ist- Konstruktionen und Extrapositionen mit es. Gesprächsforschung - Online-Zeitschrift zur verbalen Interaktion 9, 86-114.

Günthner, Susanne (2011): Grammatical constructions and communicative genres. In Heidrun Dorgeloh \& Anja Wanner (Hrsg.), Syntactic Variation and Genre, 195-217. Berlin, Boston: De Gruyter.

Günthner, Susanne (2013): Vom schriftsprachlichen Standard zur pragmatischen Vielfalt? Aspekte einer interaktional fundierten Grammatikbeschreibung am Beispiel von dassKonstruktionen. In Jörg Hagemann, Wolf P. Klein \& Sven Staffeldt (Hrsg.), Pragmatischer Standard, 223-243. Tübingen: Stauffenburg.

Günthner, Susanne (2014): The dynamics of dass-constructions in everyday German interactions - a dialogical perspective. In Susanne Günthner, Wolfgang Imo \& Jörg Bücker (Hrsg.), Grammar and Dialogism. Sequential, Syntactic, and Prosodic Patterns between Emergence and Sedimentation, 179-206. Berlin, Boston: De Gruyter.

Heritage, John (2013): Epistemics in Conversation. In Jack Sidnell \& Tanya Stivers (Hrsg.), The Handbook of Conversation Analysis, 370-394. Malden u. a.: Blackwell.

Hewitt, John P. \& Randall Stokes (1975): Disclaimers. American Sociological Review 40 (1), 1-11.

Imo, Wolfgang (2015): Was ist (k)eine Konstruktion? In Christa Dürscheid \& Jan G. Schneider (Hrsg.), Handbuch Satz, Äußerung, Schema. Berlin, Boston: De Gruyter.

Linell, Per (2005): The Written Language Bias in Linguistics: Its Nature, Origins and Transformations. London, New York: Routledge.

Lukes, Steven (1978): Power and authority. In Tom Bottomore \& Robert Nisbet (Hrsg.), A History of Sociological Analysis, 633-676. New York: Basic Books.

Oloff, Florence (2017): Genau als redebeitragsinterne, responsive, sequenzschließende oder sequenzstrukturierende Bestätigungspartikel im Gespräch. In Hardarik Blühdorn, Arnulf Deppermann, Henrike Helmer \& Thomas Spranz-Fogasy (Hrsg.), Diskursmarker im Deutschen. Reflexionen und Analysen, 207-232. Göttingen: Verlag für Gesprächsforschung. 
Oppenrieder, Wilhelm (1991): Von Subjekten, Sätzen und Subjektsätzen. Untersuchungen zur Syntax des Deutschen. Tübingen: Niemeyer.

Reis, Marga (1997): Zum syntaktischen Status unselbstständiger Verbzweit-Sätze. In Christa Dürscheid, Karl-Heinz Ramers \& Monika Schwarz (Hrsg.), Sprache im Fokus. Festschrift für Heinz Vater, 121-144. Tübingen: Niemeyer.

Schegloff, Emanuel A. (1996): Confirming Allusions: Toward an Empirical Account of Action. American Journal of Sociology 102, 161-216.

Schlobinski, Peter (1992): Funktionale Grammatik und Sprachbeschreibung. Eine Untersuchung zum gesprochenen Deutsch sowie zum Chinesischen. Opladen: Westdeutscher Verlag.

Selting, Margret (2007): Lists as embedded structures and the prosody of list construction as an interactional resource. Journal of Pragmatics 39, 483-526.

Selting, Margret, Peter Auer, Dagmar Barth-Weingarten, Jörg R. Bergmann, Pia Bergmann, Karin Birkner, Elizabeth Couper-Kuhlen, Arnulf Deppermann, Peter Gilles, Susanne Günthner, Martin Hartung, Friederike Kern, Christine Mertzlufft, Christian Meyer, Miriam Morek, Frank Oberzaucher, Jörg Peters, Uta Quasthoff, Wilfried Schütte, Anja Stukenbrock \& Susanne Uhmann (2009): Gesprächsanalytisches Transkriptionssystem 2. Gesprächsforschung - Online-Zeitschrift zur verbalen Interaktion 10, 353-402.

Stevanovic, Melisa \& Anssi Peräkylä (2012): Deontic Authority in Interaction: The Right to Announce, Propose, and Decide. Research on Language \& Social Interaction 45 (3), 297-321.

Stivers, Tanya, John Heritage, Rebecca K Barnes, Rose McCabe, Laura Thompson \& Merran Toerien (2018): Treatment Recommendations as Actions. Health Communication 33 (11), $1335-1344$.

Tannen, Deborah (2007): Talking Voices. Repetition, Dialogue, and Imagery in Conversational Discourse. Cambridge: Cambridge University Press.

Weuster, Edith (1983): Nicht-eingebettete Satztypen mit Verb-Endstellung im Deutschen. In Klaus Olszok \& Edith Weuster (Hrsg.), Zur Wortstellungsproblematik im Deutschen, 7-87. Tübingen: Narr.

Zifonun, Gisela, Ludger Hoffmann \& Bruno Strecker (1997): Grammatik der deutschen Sprache. Berlin u. a.: De Gruyter. 